\title{
Association Between RBC Antigen Allo-Antibodies and Immune-Related Adverse Events During Immune Checkpoint Inhibitor Treatment for Advanced Cancers
}

This article was published in the following Dove Press journal:

Cancer Management and Research

\author{
Natasha $A$ Jain $\mathbb{D}^{1,2}$ \\ Songzhu Zhao ${ }^{3}$ \\ Lai Wei iD $^{3}$ \\ Kerry A Rogers (iD) ${ }^{1,2}$ \\ Gregory A Otterson' \\ Tzu-Fei Wang (D) ${ }^{2}$ \\ Dwight $\mathrm{H}$ Owen (D) \\ 'Division of Medical Oncology, The Ohio \\ State University, Columbus, OH, USA; \\ ${ }^{2}$ Division of Hematology, The Ohio State \\ University, Columbus, OH, USA; ${ }^{3}$ Center \\ for Biostatistics, The Ohio State \\ University, Columbus, $\mathrm{OH}$, USA
}

Correspondence: Dwight $\mathrm{H}$ Owen Division of Medical Oncology, The Ohio State University, Starling Loving Hall, 320 West 10th Avenue, Columbus, $\mathrm{OH}$ 43212, USA

Tel + I 6I4-685-2039

Email Dwight.owen@osumc.edu
Introduction: Immune checkpoint inhibitors (ICI) have become a primary treatment modality for patients with a variety of malignancies. Given their increasing use, it is essential to be familiar with their immune-related adverse events (irAEs). Here we report a severe case of autoimmune hemolytic anemia (AIHA) associated with cold agglutinin precipitated by pembrolizumab, and a retrospective study of patients treated with ICI utilizing an institutional database where we analyzed the patterns of anti-RBC testing and their ability to predict irAE.

Methods: Patients treated with at least one dose of ICI (PD-1, PD-L1, CTLA-4 inhibitors) for advanced cancer between November 2012 and September 2017 at our institution were included. Electronic Medical Records were reviewed to abstract data. Medians and $95 \%$ CIs were estimated using Kaplan-Meier method and differences compared using the Log Rank test. Fisher's exact test and Chi square test were used to analyze clinical associations.

Results: We identified 1065 patients who received at least one dose of ICI: 180/1065 (17\%) underwent direct antiglobulin test (DAT) or allo-antibody (alloAb) testing at any time; 127/1065 $(12 \%)$ had either DAT or alloAb testing pre-ICI; 129 had either DAT or alloAb testing after ICI initiation; and 76 had either DAT or alloAb testing at both time points. There was a significant association between positive alloAb pre-ICI and the development of irAE while on ICI $(p=0.04)$. Conclusion: Given the increasing use of ICI, oncologists should be aware of potential irAEs with ICI. We found an association between the presence of an alloAb pre-ICI and the development of irAE, indicating that this previous non-self antigen response may predict immune adverse events. A larger prospective study is needed for systematic evaluation of the association between alloAb testing and irAE, and whether routine testing may inform clinical decision-making for patients.

Keywords: autoimmune hemolytic anemia, immune-related adverse events, direct antiglobulin test, immunotherapy, immune checkpoint inhibitors, red blood cell alloantibodies

\section{Background}

Immune checkpoint inhibitors (ICI) have become a primary treatment modality for patients with a broad variety of cancers, resulting in significantly prolonged survival in some patients. ${ }^{1-3}$ Given the increasing use of these agents, it is essential to be aware of potential immune-related adverse events (irAEs). The most common irAEs involve the skin, gastrointestinal tract, thyroid gland, liver, lung, and joints. ${ }^{4}$ Although less common, autoimmune hemolytic anemia (AIHA) has been reported. ${ }^{5-10}$ 
AIHA is an uncommon disorder characterized by hemolysis mediated by auto-antibodies directed against red blood cell (RBC) antigens. The incidence of AIHA as a primary disorder is $1-3$ per 100,000 per year, with a mortality rate of approximately $11 \% .{ }^{11-13}$ It is classified as either warm AIHA or cold agglutinin (CA) type by the temperature at which autoantibodies bind optimally to the RBCs. ${ }^{11}$ Warm AIHA comprises about $75 \%$ of all cases and is characterized by IgG-mediated extravascular hemolysis that occurs at $37^{\circ} \mathrm{C} .{ }^{14,15} \mathrm{CA}$ comprises about $15 \%$ of all cases and is characterized by complement-mediated hemolysis that occurs at an optimal temperature of $3-4^{\circ} \mathrm{C}$, but can occur at highertemperatures. ${ }^{16}$ The pathogenesis of AIHA associated with ICI has not been fully elucidated, but is likely to be related to direct activation of autoreactive $\mathrm{T}$ and B-cells, as well as suppression of T-regulatory (Treg) cells which may be suppressing an existing autoimmune response. ${ }^{6,18,19}$

Clinically there are two types of anti-RBC antibodies routinely tested for: auto-antibodies, and alloantibodies (alloAb) which target non-self RBC. If antibodies are present on the surface of RBCs, i.e. autoantibodies, they can be detected with a direct antiglobulin test (DAT). ${ }^{17,20}$ Immune checkpoint inhibitors are associated with the development of autoimmunity. ${ }^{6,8-10}$ AlloAb, on the other hand, are triggered by immune responses after exposure to non-self-antigens, leading to the production of antibodies against certain $\mathrm{RBC}$ antigens from another individual (after blood transfusion) or fetus (after pregnancy), which can cause destruction of donor RBC and hemolytic transfusion reactions. The presence of these antibodies is determined by an antibody screen routinely done by the blood bank prior to RBC transfusion. ${ }^{20,21}$

RBC autoimmunization may occur even without a history of transfusions in AIHA, possibly due to viral or drug-induced modification of the immune system and alteration in the $\mathrm{T}$ cell balance. $^{22}$

The association of RBC antibodies with hematologic or non-hematologic toxicity in patients treated with ICI has not been evaluated in detail. Here we report a case of hemolysis from a pre-existing CA after initiation of treatment with the PD-1 monoclonal antibody pembrolizumab. This experience led us to conduct a retrospective study to evaluate the association of baseline RBC antibodies and development of irAE.

\section{Case Presentation}

A 47-year-old woman with history of tobacco use was found to have a cavitary lung mass and a progressively enlarging thigh mass with encasement of vascular structures but no osseous invasion. Subsequent biopsy revealed poorly differentiated carcinoma with spindle cell features. PDL-1 expression was $99 \%$ as assessed by tumor proportion score using the $22 \mathrm{C} 3$ antibody and she was diagnosed with metastatic non-small cell lung cancer (NSCLC). Blood-based tumor next-generation sequencing showed mutational profile of KRAS and TP53, supportive of the diagnosis.

Pembrolizumab as first-line therapy was planned given the high expression of PD-L1 (99\%) in line with the KEYNOTE-24 trial. ${ }^{2}$ However, prior to treatment initiation, she developed severe anemia with hemoglobin $(\mathrm{Hb})$ of $6 \mathrm{~g} / \mathrm{dL}$, which prompted an anemia workup and was significant for a positive DAT with complement $\mathrm{C} 3$ but not IgG. Cold agglutinin titer was positive with titer of 64 (normal: $<3$ at 4 degree Celsius). There was no evidence of hemolysis with elevated haptoglobin (381, normal 44-215 mg/dL), normal total bilirubin (0.8, normal $<1.5 \mathrm{mg} / \mathrm{dL})$, and absolute reticulocyte count $(0.05$, nor$\mathrm{mal} 0.01-0.07 \mathrm{M} / \mathrm{uL}$ ) that was inappropriate for the degree of anemia. Lactate dehydrogenase (LDH) was only slightly elevated (236, normal 100-190 U/L). Flow cytometry of peripheral blood and monoclonal protein workup was unremarkable. Peripheral smear showed agglutinating RBC. The patient's positive cold agglutinin titer was thought to be secondary to her underlying lung cancer and not associated with hemolysis at this time, and initiation of treatment for the malignancy was recommended. She received transfusion with improvement in $\mathrm{Hb}$ to 8.5 $\mathrm{g} / \mathrm{dL}$.

The patient was started on pembrolizumab monotherapy. $\mathrm{Hb}$ subsequently dropped to $6.1 \mathrm{~g} / \mathrm{dL}$ after the second cycle of pembrolizumab requiring multiple RBC transfusions. At this time, she demonstrated evidence of hemolysis: LDH increased to $396 \mathrm{U} / \mathrm{L}$ (normal 100-190), haptoglobin now became undetectable, and indirect bilirubin was elevated to $1.1 \mathrm{mg} / \mathrm{dL}$. In addition, her platelet count decreased from 126 to 81 (x 10\%/L) within one week. It was therefore thought that she had developed immunemediated hemolysis from CA syndrome. A trial of corticosteroids (prednisone $1 \mathrm{mg} / \mathrm{kg}$ daily) was given as this occurred after ICI despite this not being a standard initial treatment for $\mathrm{CA}$ syndrome. She had improvement in 
hematologic parameters after this with her platelet count increasing to 173 ( $10^{9} / \mathrm{L}$ ) and hemoglobin to $12.5 \mathrm{~g} / \mathrm{dL}$. No further blood transfusion was needed. Repeat staging scans showed progression of the lung cancer. However, before she could receive next line treatment she developed massive hemorrhage from tumor invasion into the right superficial femoral artery and died from major hemorrhage.

This case of CA syndrome from existing CA worsened by pembrolizumab revealed the importance of understanding irAE associated with ICI and prompted the following retrospective study to further investigate irAE in patients treated with ICI. We also looked at the antibody screens as these were readily available (being done as a part of the routine blood bank work up prior to transfusions).

\section{Methods}

To evaluate the association between baseline anti-RBC testing, with both DAT and antibody screen for alloAb, and the development of irAE in patients treated with ICI, we identified a cohort of 1065 adult patients treated with ICI, either as a standard of care or on a clinical trial, for any advanced malignancy between November 2012 and September 2017. Patients of at least 18 years of age at the time of ICI were included. There were no exclusion criteria. Documentation of receipt of ICI was confirmed using pharmacy administration database. Electronic Medical Records were reviewed to extract data. Patient demographics, disease characteristics (stage, histology), treatment received (ICI), and toxicity or irAE were all collected in a REDCap database. ${ }^{23}$ The study was approved by the institutional review board at the Ohio State University Comprehensive Cancer Center.

\section{Statistical Analysis}

Patient characteristics were summarized using descriptive statistics including medians and interquartile for the continuous variables and frequencies for the categorical variables. These characteristics have been compared between patients with and without pre-ICI testing using Chi-square test for the categorical variables and Kruskal-Wallis for the continuous variables. Overall Survival (OS) was calculated from the date of initiation of ICI to death from any cause or last follow up. Median OS with 95\% confidence intervals was estimated using Kaplan-Meier method. OS was compared between patients with and without Pre-ICI positive DAT or alloAb. The association between irAE and DAT or alloAb testing was evaluated using Fisher's exact test.

\section{Results}

\section{Patient Characteristics}

A total of 1065 patients treated with ICI between November 2012 and September 2017 were identified. Among them, 127 patients had either DAT or alloAb testing prior to ICI initiation (pre-ICI testing). Patient characteristics of the entire cohort $(n=1065)$ compared to those with pre-ICI testing $(n=127)$ as well as those without pre-ICI testing (n-938) are shown in Table 1. There was no difference in age between the two groups $(p=0.28)$. Seventy-six (7\%) patients in the entire cohort (1065), and $17(14 \%)$ among the 127 patients had either DAT or alloAb testing prior to ICI initiation (pre-ICI testing). Compared to the entire cohort, significantly more females $(\mathrm{p}=0.02)$, more smokers $(\mathrm{p}=<0.0001)$, more patients with NSCLC $(\mathrm{p}=<0.0001)$, and more patients who ultimately recieved PD1/PD-L1 monotherapy, PD1/CTLA4, and CTLA4 monotherapy $(\mathrm{p}=<0.0001)$ underwent pre-ICI testing.

\section{DAT or alloAb Testing}

One hundred and eighty out of 1065 patients had either DAT or alloAb testing at any time (Supplementary Figure 1). Of these 180 patients, 127 had either DAT or alloAb testing pre-ICI; 129 had either DAT or alloAb testing after ICI initiation; and 76 had either DAT or alloAb testing at both time points.

We identified seven patients who had a positive DAT either pre-ICI or post-ICI. Of these, five patients had a positive DAT pre-ICI and two did not. We identified three patients with a positive DAT after ICI treatment. One of these three patients had a positive testing pre-ICI, and the other two did not have pre-ICI testing. Outcomes of these patients with positive DAT at either pre-ICI or post- ICI are shown in Table 2.

\section{Associations of Clinical Outcomes}

Among the 127 patients who had either DAT or alloAb testing prior to ICI initiation, there was no association between presence of positive DAT at pre-ICI and development of irAEs $(p=0.10)$. The irAE rate was for patients with pre-ICI alloantibodies (4 of 6) was significantly 
Table I Patient Characteristics

\begin{tabular}{|c|c|c|c|c|c|}
\hline & & $\begin{array}{l}\text { Entire Cohort } \\
N=1065\end{array}$ & $\begin{array}{l}\text { Patients with Pre-ICI } \\
\text { Testing } * N=127\end{array}$ & $\begin{array}{l}\text { Patients without Pre-ICI } \\
\text { Testing }{ }^{\#} \mathbf{N}=938\end{array}$ & p-value \\
\hline $\begin{array}{l}\text { Age, median } \\
\text { (range) }\end{array}$ & & 61.4 (19.4-92) & $61.9(29.6-91.7)$ & $61.3(19.4-92)$ & 0.2883 \\
\hline Female, n (\%) & & $437(4 I)$ & $64(50)$ & $373(40)$ & 0.0223 \\
\hline Race, n (\%) & $\begin{array}{l}\text { Caucasian } \\
\text { Non-Caucasian } \\
\text { Unknown }\end{array}$ & $\begin{array}{l}981(92) \\
82(8) \\
2\end{array}$ & $\begin{array}{l}109(86) \\
18(14) \\
0\end{array}$ & $\begin{array}{l}872(93) \\
64(7) \\
2\end{array}$ & 0.0003 \\
\hline $\begin{array}{l}\text { Cancer types, } \\
\text { n (\%) }\end{array}$ & $\begin{array}{l}\text { NSCLC } \\
\text { Melanoma } \\
\text { RCC } \\
\text { Head and neck } \\
\text { Others }{ }^{\circ}\end{array}$ & $\begin{array}{l}197(19) \\
341(32) \\
117(11) \\
65(6) \\
342(32)\end{array}$ & $\begin{array}{l}96(76) \\
5(4) \\
8(6) \\
0(0) \\
18(14)\end{array}$ & $\begin{array}{l}101(11) \\
336(36) \\
109(12) \\
68(7) \\
324(35)\end{array}$ & $<0.0001$ \\
\hline $\begin{array}{l}\text { Smoking } \\
\text { status, n (\%) }\end{array}$ & $\begin{array}{l}\text { Yes } \\
\text { No } \\
\text { Missing }\end{array}$ & $\begin{array}{l}625(59) \\
436(4 I) \\
4\end{array}$ & $\begin{array}{l}102(81) \\
24(19) \\
1\end{array}$ & $\begin{array}{l}523(56) \\
412(44) \\
3\end{array}$ & $<0.0001$ \\
\hline ICI type, n (\%) & $\begin{array}{l}\text { PDI/PDLI } \\
\text { monotherapy } \\
\text { PDI/CTLA4 } \\
\text { CTLA4 } \\
\text { monotherapy } \\
\text { Missing } \\
\text { Others }{ }^{*}\end{array}$ & $\begin{array}{l}755(74) \\
76(7) \\
193(19) \\
41 \\
234(22)\end{array}$ & $\begin{array}{l}104(86) \\
17(14) \\
0(0) \\
6 \\
6(5)\end{array}$ & $\begin{array}{l}651(72) \\
59(7) \\
193(2 \mathrm{I}) \\
35 \\
228(24)\end{array}$ & $<0.0001$ \\
\hline
\end{tabular}

Notes: *Baseline direct antiglobulin testing or allo-antibody testing. ${ }^{*}$ No baseline direct antiglobulin testing or allo-antibody testing. Others ${ }^{\Pi}$ - bladder cancer, breast cancer, Hodgkin Lymphoma, melanoma, Merkle cell carcinoma, pancreatic cancer, prostate cancer, sarcoma, small cell lung cancer. Others ${ }^{*}$ - immune checkpoint inhibitor combinations (chemotherapy and targeted therapy); and novel immune checkpoint inhibitor agents as part of clinical trials. Percentages may not add up to I00\% as some patients received more than one type of treatment. Numbers in bold indicate significant $p$-values

Abbreviations: CTLA4, cytotoxic T-lymphocyte-associated protein 4; ICl, immune checkpoint inhibitors; NSCLC, non-small cell lung cancer; PDI, programmed cell death protein I; PDLI, programmed cell death receptor ligand I; RCC, renal cell carcinoma; SCLC, small cell lung cancer.

Table 2 Outcomes of Patients with Positive DAT at Either Pre-ICl or Post-ICl

\begin{tabular}{|c|c|c|c|c|c|c|c|c|c|}
\hline $\begin{array}{l}\text { Pt } \\
\text { No. }\end{array}$ & Cancer & $\begin{array}{l}\text { Type of } \\
\text { Treatment }\end{array}$ & $\begin{array}{l}\text { Age } \\
\text { at ICI }\end{array}$ & Sex & $\begin{array}{l}\text { DAT Pre- } \\
\text { ICI }\end{array}$ & $\begin{array}{l}\text { Pattern of } \\
\text { DAT Pre ICI }\end{array}$ & $\begin{array}{l}\text { DAT } \\
\text { Post-ICI }\end{array}$ & $\begin{array}{l}\text { Pattern of } \\
\text { DAT Post ICI }\end{array}$ & $\begin{array}{l}\text { Clinically } \\
\text { Significant } \\
\text { AlHA }\end{array}$ \\
\hline I & Gastric Cancer & Nivolumab & 59 & $M$ & Positive & Anti-lgG + C3- & Not tested & $\mathrm{N} / \mathrm{A}$ & $\mathrm{N} / \mathrm{A}$ \\
\hline 2 & NSCLC & Nivolumab & 61 & $M$ & Positive & Anti-lgG + C3- & Not tested & $\mathrm{N} / \mathrm{A}$ & $\mathrm{N} / \mathrm{A}$ \\
\hline 3 & Breast Cancer & Nivolumab & 41 & $\mathrm{~F}$ & Positive & Anti-lgG $+C 3+$ & Not tested & $\mathrm{N} / \mathrm{A}$ & $\mathrm{N} / \mathrm{A}$ \\
\hline 4 & NSCLC & $\begin{array}{l}\text { Nivolumab+ } \\
\text { Ipilimumab }\end{array}$ & 57 & $M$ & Positive & Anti-lgG + C3+ & Not tested & $\mathrm{N} / \mathrm{A}$ & $\mathrm{N} / \mathrm{A}$ \\
\hline 5 & $\mathrm{RCC}$ & Nivolumab & 73 & $\mathrm{~F}$ & Positive & Anti-lgG + C3- & Positive & Anti-lgG + C3 - & Possible \\
\hline 6 & NSCLC & Nivolumab & 65 & $M$ & Not tested & N/A & Positive & Anti-lgG+C3- & No \\
\hline 7 & $\mathrm{HL}$ & Nivolumab & 34 & $\mathrm{~F}$ & Not tested & N/A & Positive & Anti-lgG $+\mathrm{C} 3+$ & Yes \\
\hline
\end{tabular}

Abbreviations: +, positive; -, negative; AlHA, autoimmune hemolytic anemia; DAT, direct antiglobulin test; $\mathrm{F}$, female; HL, Hodgkin lymphoma; ICl, immune checkpoint inhibitors; M, male; No, number; N/A, not applicable; NSCLC, non-small cell lung cancer; pre-ICl, pre-immune checkpoint inhibitors; RCC, renal cell carcinoma.

higher than patient without pre-ICI alloantibodies $(67 \%$ vs $24 \%, \mathrm{p}=0.04)$. Table 3 shows the characteristics of the six patients with alloAb pre-ICI. Four of these six patients developed an irAE affecting either skin, lung or thyroid (non-hematologic irAE). The median OS for the entire cohort was 7.6 months (range: 5.8-9.9). Pre-ICI positive DAT or alloAb at pre-ICI did not affect OS $(\mathrm{p}=0.17$ and $\mathrm{p}=0.32$, respectively). 
Table 3 Characteristics of Six Patients with Presence of alloAb Pre-ICl and Development of irAE

\begin{tabular}{|l|l|l|l|l|l|}
\hline Patient Number & Cancer & Age at ICI & Sex & irAE & Type of irAE \\
\hline $\mathbf{I}$ & NSCLC & 63 & Male & No & N/A \\
$\mathbf{2}$ & CLL, SCC kin & 85 & Male & Yes & Pneumonitis \\
$\mathbf{3}$ & HL & 53 & Female & No & N/A \\
$\mathbf{4}$ & Gastric cancer & 59 & Male & Yes & Thyroid abnormalities \\
$\mathbf{5}$ & RCC & 73 & Female & Yes & Dermatitis, pneumonitis \\
$\mathbf{6}$ & NSCLC & 57 & Male & Yes & Thyroid abnormalities, dermatitis \\
\hline
\end{tabular}

Abbreviations: alloAb, allo-antibody; CLL, chronic lymphocytic leukemia; HL, Hodgkin lymphoma; ICI, immune checkpoint inhibitor; irAE, immune-related adverse event; N/A, not applicable; NSCLC, non-small cell lung cancer; pre-ICl, pre-immune checkpoint inhibitors; RCC, renal cell carcinoma; SCC, squamous cell cancer.

\section{Discussion}

We report a case of presumed malignancy-associated cold agglutinin titer who developed hemolytic anemia after ICI therapy. The patient responded to corticosteroids, confirming the immune-mediated nature of the hemolysis. In the literature, most reported cases of hemolytic anemia after ICI were warm AIHA with a positive DAT for IgG; ${ }^{5-9}$ but one case of $\mathrm{C} 3$ positive hemolysis was reported. ${ }^{10}$ The case prompted our retrospective study to evaluate irAE including AIHA in patients receiving ICI.

First, in our study, we found a low rate of RBC antibody workup prior to initiating ICI. Out of the 1065 ICI treated patients in the entire cohort, only $127(12 \%)$ had either a DAT or alloAb testing prior to starting ICI and 180 $(17 \%)$ had either a DAT or alloAb testing at any time. In the cancer population, cytopenias including anemia and thrombocytopenia are commonly attributed to advanced malignancy and/or concurrent cancer therapies, which could lead to under-recognition and under-diagnosis of cytopenia as an irAE (such as AIHA). Given the increasing use of ICI, we believe that increasing clinical vigilance to potential irAE is necessary to monitor for these toxicities, and risk factors need to be elucidated. Currently, there are no reported biomarkers to predict the development of irAEs. Therefore, identifying such tests, if available, to help predict the development of irAE would be useful. ${ }^{24,25}$

In our cohort, we did not find an association between positive autoantibodies and the development of irAE. However, we acknowledged that our number of patients with pre-ICI testing was small and likely had insufficient power. Therefore, we cannot rule out the possibility that an association would exist if a larger sample size is available. Cooling et al previously reported two patients who developed a positive DAT during ICI treatment, and although they did not have evidence of hemolysis, both developed additional non-hematologic irAE later. $^{26}$
Interestingly, despite the small sample size, we found a significant association between positive alloAb, not autoantibodies, at pre-ICI and the development of irAE. This finding was unexpected and the etiologies remain unclear. It could be because of selection bias that the detection of alloAb indicated the selection of a "sicker population" who required blood transfusion (as blood bank workup was mostly prompted by the need of transfusion in this retrospective study). However, it might also be due to differences in the immune status of those who develop alloAb. The possible mechanisms may be inferred from other common ipilimumab-associated irAEs studies. ${ }^{27}$ Treg identified as CD4+ FOXP3+ T cells produce IL-10 as a way to suppress effector $\mathrm{T}$ cells and thus prevent autoimmunity. ${ }^{28}$ Anti-CTLA4 (cytotoxic T-lymphocyteassociated protein 4) causes loss of Treg, which is thought to cause irAE, including immune cytopenias and AIHA. ${ }^{29-31}$ Other mechanisms proposed include elevated Th1- and Th17-mediated immune responses resulting in elevated circulating levels of IL-17 and IFN- $\gamma,{ }^{32}$ increased $T$ cell activity against self-antigens, and enhanced complement-mediated inflammation. ${ }^{33}$

The strength of our study is to include more than 1000 patients with a variety of cancers as well as variety of ICI treatment regimens. However, there are some notable limitations. First, this is a retrospective study, and as such the decision to test for alloAb and/or DAT was determined by clinicians without a standard testing protocol. Therefore, a selection bias may exist in the patients who had antibody testing done which could be a sicker population needing transfusion (many patients had the RBC antibody testing done only prior to blood transfusion). Our study did reveal the low rate of RBC antibody work up and demonstrated the concern for low index of suspicion in this population which can be improved upon, as anemia was commonly attributed to advanced malignancy and/or multiple lines of pretreatment with chemotherapies. We had a small sample 
size of patients with pre-ICI testing; therefore, it is possible that an association between DAT and irAE was missed due to insufficient power. Lastly, given that antibody testing was not done systematically, we were not able to draw definitive conclusions about the true incidence of AIHA in this cohort pre-ICI. We found an association between the presence of Alloab and development of irAE, but our study also could not elucidate the pathophysiology of this association or whether a history of AIHA would preclude patients from use of ICI. These questions warrant further investigation.

\section{Conclusions}

In our study, we found a low rate of RBC antibody testing. While we did not find a significant association between presence of positive DAT pre-ICI and development of irAEs, we found an association between pre-ICI alloAb and development of non-hematologic irAE. While limited by small sample size, our findings are hypothesis generating. Future studies could consider the incorporation of systemic evaluation of antibody testing in a larger cohort to provide further information to assist in the optimal management in this patient population.

\section{Ethical Considerations}

Patient provided written consent for publication of the case details as part of OSU Total Cancer Care Protocol (OSU IRB\#2013H0197)

\section{Author Contributions}

All authors made a significant contribution to the work reported, whether that is in the conception, study design, execution, acquisition of data, analysis and interpretation, or in all these areas; took part in drafting, revising or critically reviewing the article; gave final approval of the version to be published; have agreed on the journal to which the article has been submitted; and agree to be accountable for all aspects of the work.

\section{Funding}

Research reported in this publication was supported in part by the Ohio State University Comprehensive Cancer Center and the National Institutes of Health under grant number P30 CA016058. Dr. Owen is supported by a Paul Calabresi Career Development Award for Clinical Oncology K12CA133250. Dr. Rogers is a Scholar in Clinical Research of The Leukemia \& Lymphoma Society (CDP 2331-20).

\section{Disclosure}

Kerry Rogers reports grants from Genetech, AbbVie, and Janssen and personal fees from Acerta Pharma, AstraZeneca, and Pharmacyclics, during the conduct of the study.

Dwight Owen reports grants from Palobiofarma, BMS, Merck, and Genentech and personal fees from theMednet. org and AstraZeneca, outside the submitted work.

The authors report no other potential conflicts of interest for this work.

\section{References}

1. Motzer RJ, Tannir NM, McDermott DF, et al. Nivolumab plus ipilimumab versus sunitinib in advanced renal-cell carcinoma. $N$ Engl J Med. 2018;378(14):1277-1290. doi:10.1056/ NEJMoa1712126

2. Reck M, Rodriguez-Abreu D, Robinson AG, et al. Pembrolizumab versus chemotherapy for PD-L1-positive non-small-cell lung cancer. $N$ Engl J Med. 2016;375(19):1823-1833. doi:10.1056/NEJMoa 1606774

3. Ferris RL, Blumenschein G Jr, Fayette J, et al. Nivolumab for recurrent squamous-cell carcinoma of the head and neck. $N$ Engl J Med. 2016;375(19):1856-1867. doi:10.1056/NEJMoa1602252

4. Delanoy N, Michot JM, Comont $\mathrm{T}$, et al. Haematological immune-related adverse events induced by anti-PD-1 or anti-PD-L1 immunotherapy: a descriptive observational study. Lancet Haematol. 2019;6(1):e48-e57. doi:10.1016/S2352-3026(18)30175-3

5. Nair R, Gheith S, Nair SG. Immunotherapy-associated hemolytic anemia with pure red-cell aplasia. $N$ Engl J Med. 2016;374 (11):1096-1097. doi:10.1056/NEJMc1509362

6. Kong BY, Micklethwaite KP, Swaminathan S, Kefford RF, Carlino MS. Autoimmune hemolytic anemia induced by anti-PD-1 therapy in metastatic melanoma. Melanoma Res. 2016;26 (2):202-204. doi:10.1097/CMR.0000000000000232

7. Ogawa K, Ito J, Fujimoto D, et al. Exacerbation of autoimmune hemolytic anemia induced by the first dose of programmed death-1 inhibitor pembrolizumab: a case report. Invest New Drugs. 2018;36 (3):509-512. doi:10.1007/s10637-018-0561-5

8. Schwab KS, Heine A, Weimann T, Kristiansen G, Brossart P. Development of hemolytic anemia in a nivolumab-treated patient with refractory metastatic squamous cell skin cancer and chronic lymphatic leukemia. Case Rep Oncol. 2016;9(2):373-378. doi: $10.1159 / 000447508$

9. Tardy MP, Gastaud L, Boscagli A, Peyrade F, Gallamini A, Thyss A. Autoimmune hemolytic anemia after nivolumab treatment in Hodgkin lymphoma responsive to immunosuppressive treatment. A case report. Hematol Oncol. 2017;35(4):875-877. doi:10.1002/ hon. 2338

10. Palla AR, Kennedy D, Mosharraf H, Doll D. Autoimmune hemolytic anemia as a complication of nivolumab therapy. Case Rep Oncol. 2016;9(3):691-697. doi:10.1159/000452296

11. Lechner K, Jager U. How I treat autoimmune hemolytic anemias in adults. Blood. 2010;116(11):1831-1838. doi:10.1182/blood-2010-03259325

12. Zanella A, Barcellini W. Treatment of autoimmune hemolytic anemias. Haematologica. 2014;99(10):1547-1554. doi:10.3324/ haematol.2014.114561

13. Jäger U, Barcellini W, Broome CM, et al. Diagnosis and treatment of autoimmune hemolytic anemia in adults: recommendations from the First International Consensus Meeting. Blood Rev. 2020;41:100648. doi:10.1016/j.blre.2019.100648 
14. Bass GF, Tuscano ET, Tuscano JM. Diagnosis and classification of autoimmune hemolytic anemia. Autoimmun Rev. 2014;13(4-5):560-564. doi:10.1016/j.autrev.2013.11.010

15. Park SH. Diagnosis and treatment of autoimmune hemolytic anemia: classic approach and recent advances. Blood Res. 2016;51(2):69-71. doi:10.5045/br.2016.51.2.69

16. Berentsen S. Cold agglutinin disease. Hematology Am Soc Hematol Educ Program. 2016;2016(1):226-231. doi:10.1182/asheducation2016.1.226

17. Mqadmi A, Zheng X, Yazdanbakhsh K. CD4+CD25+ regulatory $\mathrm{T}$ cells control induction of autoimmune hemolytic anemia. Blood. 2005;105(9):3746-3748. doi:10.1182/blood-2004-12-4692

18. Barcellini W. New insights in the pathogenesis of autoimmune hemolytic anemia. Transfus Med Hemother. 2015;42(5):287-293. doi:10.1159/000439002

19. Berentsen S. How I manage cold agglutinin disease. Br J Haematol. 2011;153(3):309-317. doi:10.1111/j.1365-2141.2011.08643.x

20. Tormey CA, Hendrickson JE. Transfusion-related red blood cell alloantibodies: induction and consequences. Blood. 2019;133 (17):1821-1830. doi:10.1182/blood-2018-08-833962

21. Singer ST, Wu V, Mignacca R, Kuypers FA, Morel P, Vichinsky EP. Alloimmunization and erythrocyte autoimmunization in transfusion-dependent thalassemia patients of predominantly asian descent. Blood. 2000;96(10):3369-3373. doi:10.1182/blood.V96.10.3 369

22. Oliveira MC, Oliveira BM, Murao M, Vieira ZM, Gresta LT, Viana MB. Clinical course of autoimmune hemolytic anemia: an observational study. J Pediatr (Rio J). 2006;82(1):58-62.

23. Harris PA, Taylor R, Thielke R, Payne J, Gonzalez N, Conde JG. Research electronic data capture (REDCap)-a metadata-driven methodology and workflow process for providing translational research informatics support. $J$ Biomed Inform. 2009;42(2):377-381. doi:10.1016/j.jbi.2008.08.010

24. Nuzzo PV, Pond GR, Abou Alaiwi S, et al. Conditional immune toxicity rate in patients with metastatic renal and urothelial cancer treated with immune checkpoint inhibitors. J Immunother Cancer. 2020;8(1):e000371. doi:10.1136/jitc-2019-000371
25. Owen DH, Wei L, Bertino EM, et al. Incidence, risk factors, and effect on survival of immune-related adverse events in patients with non-small-cell lung cancer. Clin Lung Cancer. 2018;19(6):e893e900. doi:10.1016/j.cllc.2018.08.008

26. Cooling LL, Sherbeck J, Mowers JC, Hugan SL. Development of red blood cell autoantibodies following treatment with checkpoint inhibitors: a new class of anti-neoplastic, immunotherapeutic agents associated with immune dysregulation. Immunohematology. 2017;33 (1):15-21.

27. Weinmann SC, Pisetsky DS. Mechanisms of immune-related adverse events during the treatment of cancer with immune checkpoint inhibitors. Rheumatology. 2019;58(Supp17):vii59-vii67. doi:10.109 3/rheumatology/kez308

28. Sakaguchi S. Regulatory T cells: key controllers of immunologic self-tolerance. Cell. 2000;101(5):455-458. doi:10.1016/S00928674(00)80856-9

29. von Euw E, Chodon T, Attar N, et al. CTLA4 blockade increases Th17 cells in patients with metastatic melanoma. J Transl Med. 2009; 7:35. doi:10.1186/1479-5876-7-35

30. Ward FJ, Hall AM, Cairns LS, et al. Clonal regulatory T cells specific for a red blood cell autoantigen in human autoimmune hemolytic anemia. Blood. 2008;111(2):680-687. doi:10.1182/blood-2007-07101345

31. Simpson TR, Li F, Montalvo-Ortiz W, et al. Fc-dependent depletion of tumor-infiltrating regulatory $\mathrm{T}$ cells co-defines the efficacy of anti-CTLA-4 therapy against melanoma. J Exp Med. 2013;210 (9):1695-1710. doi:10.1084/jem.20130579

32. Dulos J, Carven GJ, van Boxtel SJ, et al. PD-1 blockade augments Th1 and Th17 and suppresses Th2 responses in peripheral blood from patients with prostate and advanced melanoma cancer. $J$ Immunother. 2012;35(2):169-178. doi:10.1097/CJI.0b013e318247a4e7

33. Olson DJ, Rajagopal P, Tjota MY, Venkataraman G, Luke JJ, Gajewski TF. A case of dual-mechanism immune-related anaemia in a patient with metastatic melanoma treated with nivolumab and ipilimumab. J Immunother Cancer. 2020;8(1):e000380. doi:10.1136/ jitc-2019-000380

\section{Publish your work in this journal}

Cancer Management and Research is an international, peer-reviewed open access journal focusing on cancer research and the optimal use of preventative and integrated treatment interventions to achieve improved outcomes, enhanced survival and quality of life for the cancer patient.
The manuscript management system is completely online and includes a very quick and fair peer-review system, which is all easy to use. Visit http://www.dovepress.com/testimonials.php to read real quotes from published authors. 